\title{
Donde habite el olvido
}

\section{MEI}

II, vol. 6 $\mathrm{n}^{0} 10$

\author{
Pilar del Campo Puerta \\ Doctora en Ciencias dela Documentación \\ Rafael González Sánchez \\ Licenciado en Documentación
}

Recibido el 13-07-2015

\section{Resumen}

El desarrollo de las nuevas tecnologías de la información y de la comunicación y su plena inserción en la vida personal y profesional ha traído consigo, además de un cambio en los modos de trabajo y de interrelación humana, la amplitud y/o modificación de los significados de algunos términos. El concepto de 'olvido' es uno de tantos.

Este trabajo aborda dos áreas antagónicas del olvido con respecto alas nuevas tecnologías de la información y la comunicación: el olvido como la necesidad de que 'Internet se olvide de mí, y el olvido como la amenaza que puede verse paliada apoyándose en las posibilidades que Internet aporta.

En primer lugar, se exponen los significados del término 'olvido'. A continuación y pese a ser un problema global, nos centramos principalmente en las diferentes iniciativas en el marco de la Unión Europea en general y de España en particular, que se están llevando a cabo para regular el almacenamiento de información en la red y el derecho que el ciudadano tiene para reclamar el borrado de datos personales que atenten contra su intimidad y reputación.

Por último, se analiza el estímulo terapéutico que los nuevos recursos tecnológicos pueden ofrecer para el tratamiento de enfermedades donde habita el olvido como son el Alzheimer y la amnesia.

\section{Palabras clave}

Amnesia-Tratamiento, Derecho al olvido, Enfermedad de AlzheimerTratamiento, Nuevas tecnologías-Uso terapéutico, Privacidad en internet, Protección de datos.

\section{Title}

Where oblivion inhabits

\begin{abstract}
The development of the new information and communication technologies and its full insertion in the personal and professional life has brought with it, in addition to a change in the ways of work and of human interrelation, the largeness and/or modification of the meanings of some terms. The concept of 'negligence'is one of them.

This work tackles two antagonistic areas of the negligence with regard to the new information technologies and the communication: the negligence as the need
\end{abstract}

Campo Puerta, Pilar del. González Sánchez, Rafael . "Donde habite el olvido”. En: Métodos de información (MEI), II Época, Vol. 6, no 810 2015, pp. 87-108.

DOI: http://dx.doi.o rg/10.5557/IIMEI6-N10-087108 
that 'Internet forgets me', and the negligence like the threat that can turns relieved resting on the possibilities that Internet contributes.

Firstly, the meanings of 'negligence' are exhibited. Next and despite being a global problem, we center principally on the different initiatives in the frame of the European Union in general and of Spain in particular, which is carried out to regulate the information storage in the network and a right that the citizen has to claim the erasure of personal details that commit an outrage against its intimacy and reputation.

Finally, there is analyzed the therapeutic stimulus that the new technological resources can offer for the treatment of illnesses where he inhabits the negligence as there are the Alzheimer's disease and the amnesia.

\section{KEYWORDS}

Amnesia-Treatment, The right to be forgotten,Alzheimer's disease-Treatment, New technologies-Therapeutic use, Privacy on the Internet, Data protection.

«El hombre posee el privilegio de tener antepasados; somos hijos siempre de alguien, herederos y descendientes. Mas cuando se pertenece a un mundo tan completo como el de la cultura occidental los antepasados son múltiples; tenemos diversas tradiciones detrás de nosotros, no una sola. De ahí el olvido y también los sucesivos renacimientos.

$\mathrm{Y}$ es que tener cultura, estar en una cultura, es tener detrás de la vida individual de cada uno un tesoro a veces anónimo, a veces con nombre y figura. Es poder recordar, rememorar. Poder también, en un trance difícil, aclarar en su espejo nuestra angustia e incertidumbre».

(Zambrano 2005, p. 21)

El desarrollo de las nuevas tecnologías de la información y de la comunicación y su plena inserción en la vida personal y profesional han traído consigo, además de un cambio en los modos de trabajo y de interrelación humana, la amplitud y/o modificación de los significados de algunos términos. El concepto de ‘olvido' es uno de tantos.

Desde siempre el olvido había estado vinculado a la psicología y a la medicina, en concreto, a todos los aspectos relacionados con la memoria humana y con las enfermedades que el cerebro puede padecer.

Según el Diccionario de la lengua española(Real Academia Española 2015),el olvido es la'cesación de la memoria o del afecto que se tenía', además del'descuido de algo que se debía tener presente'.

En el marco de la medicina, y más concretamente de la psiquiatría y la neurología, el olvido aparece etimológicamente vinculado a la amnesia[a(n)- 'sin' + mnēsíâ 'recuerdo'] que en griego significa 'voluntad de olvido'. La definición recogida en el Diccionario médico-biológico, histórico y etimológico (Cortés Gabaudán 2011)es 'pérdida o debilidad notable de la memoria; puede ser orgánica por lesión cerebral, enfermedad, uso de drogas; o puede ser funcional por factores psicológicos, como mecanismos de defensa'.

Ninguna vinculación ni etimológica ni conceptual relacionada hasta el momento con las nuevas tecnologías de la comunicación y de la información. Sin embargo, desde que a mediados de la década de los 90 del siglo XX y a un ritmo trepidante Internet pasó a ser una herramientamás de trabajo y de interrelación personal, otros 'entes informáticos' comenzaron 
también a instalarse en nuestra cotidianidad: Google, Youtube, Facebook, Tuenti, Yahoo!, Twitter, LinkedIn, Flickr, etc. Fue así como el mundo digital fue absorbiendo términos de otras disciplinas del conocimiento y creando una demanda de derechos que probablemente todavía no aparecen expresamente en ningún texto constitucional de cualquier país. Uno de ellos es el llamado 'derecho al olvido'.

En este trabajo vamos a abordar dos áreas antagónicas del olvido en el marco del avance tecnológico y del mundo virtual en el que nos encontramos. Por un lado, el derecho al olvido la necesidad de que 'Internet se olvide de mí'-, y por otro, el estímulo terapéutico que los nuevos recursos tecnológicos pueden ofrecer para el tratamiento de enfermedades donde habita el olvidocomo son el Alzheimer y la amnesia.

\section{EL OLVIDO COMO NECESIDAD}

\section{1 ¿Qué es el ‘derecho al olvido’?}

El 'derecho al olvido' supone la cancelación de un dato personal que se ha recabado legítimamente para que se retire cuando se agote la finalidad para la que fue obtenido. Equivale al poder de un ciudadano sin relevancia pública a disponer de toda la información personal de la que es titular y que no quiere ver su nombre ligado eternamente a esa información cuando lo teclea en un buscador, para así preservar su privacidad.Una labor que puede apreciarse titánica, a menudo frustrante, pero no imposible.

La Enciclopedia jurídicaonline-http://leyderecho.org/-define 'derecho al olvido' como la 'facultad que tiene una persona a borrar, bloquear o eliminar información personal que aparece en Internet y que de alguna forma afecte al ejercicio de alguno de sus derechos fundamentales'. Otras obras de referencia tanto en papel (Fernández Martínez 2012,Villa-Real y Arco Torres 1999,Fundación Tomás Moro 1997)como electrónicas (Diccionario jurídico de derecho de la UNED-http://www.uned-derecho.com/diccionario/-la Enciclopedia jurídica -http://www.enciclopedia-juridica.biz14.com/inicioenciclopedia-diccionario-juridico.html-, o el Diccionario jurídico-http:// diccionariojuridico.net/-, entre otros) aún no lo recogen.

La pretensión de un ciudadano de borrar los datos que hacen alusión a su persona en Internet es legítima en el momento en que su publicación no ha sido por voluntad propia, sino como consecuencia de figurar en un archivo, público o privado, y el motivo de ello carezca de interés público.Por ejemplo, personas que en su día cometieron una infracción o una falta que quedó recogida en archivos judiciales, publicada en un boletín oficial (Boletín Oficial del Estado, Diario Oficial de las Comunidades Europeas, boletín oficial de una 
región, de una provincia, etc.) o un medio de comunicación. Pero esta pretensión no es aplicable si, entre otras causas, el particular aparece en la red como autor de un delito por el que fue condenado por sentencia firme, es decir, que ya no es susceptible de recurso. Por ejemplo: Si el Dioni solicitara borrar digitalmente de su biografía que robóun furgón blindado con 298 millones de las antiguas pesetas en 1989 (Duva,Montoliu y'El Dioni' roba un furgón lleno de dinero 1989) no podría, porque fue una noticia verídica, de interés público y sobre ella cayó una sentencia judicial firme.

\subsection{El ‘derecho al olvido’ en la Unión Europea}

Aunque fue en 2006, cuando el Comité de Ministros del Consejo de Europa estableció el 28 de enero como el 'Día Europeo de la Protección de Datos', con el fin de promover $\mathrm{y}$ acercar entre los ciudadanos sus derechos $\mathrm{y}$ responsabilidades en materia de protección de datos personales, el trabajo desarrollado en este ámbito por las instituciones europeas viene de lejos. De hecho, se eligió el 28 de enero porque esta fecha corresponde al aniversario de la firma del Convenio 108 del Consejo de Europa para la protección de las personas respecto al tratamiento automatizado de datos de carácter personal (España 1985), que tuvo lugar en 1981.

\subsubsection{Directiva95/46/CE, sobreProtección de Datos}

Su nombre oficial y completo es Directiva 95/46/CE del Parlamento Europeo y del Consejo, de 24 de octubre de 1995, relativa a la protección de las personas físicas en lo que respecta al tratamiento de datos personales y a la libre circulación de estos datos (Unión Europea 1995).

Fue el primer texto legislativo europeo que reguló la protección de datos. Internet a nivel de usuario estaba gestándose y las posibilidades de comunicación, trabajo y ocio que actualmente nos dispensa nada tenían que ver con lo que por aquellos años ofrecía.

No obstante, esta Directiva fijó límites estrictos para la recogida y utilización de los datos personales y solicitaba la creación, en cada Estado miembro, de un organismo nacional independiente encargado de la protección de los mencionados datos. No cita en ningún momento el derecho al olvido, pero sí ya lo deja vislumbrar en su artículo 14 donde trata el derecho a oponerse al tratamiento de los datos de carácter personal que le conciernan.

En enero de 2012, la Comisión Europea presentó un documento que abordaba un cambio muy sustancial en la normativa sobre protección de datos personales: el Reglamento Europeo de Protección de Datos. La aprobación 
definitiva de este reglamento pondrá fin a esta Directiva.

\subsubsection{Reglamento Europeo de Protección de Datos}

En marzo del año pasado, el Parlamento Europeo (2014) "dio luz verde al proyecto del Reglamento Europeo de Protección de Datos". El 15 de junio de 2015 alcanzó su aprobación por partedel Consejo de Ministros de Justicia de los 28 países de la Unión Europea. "Las negociaciones -Parlamento Europeo, Consejo de Ministros y Comisión- siguen adelante y su aprobación definitiva para su entrada en vigorestá prevista para octubre de este año"(Parlamento Europeo 2015).

\section{A) Sus objetivos(Parlamento Europeo 2014):}

- Adaptar la protección de datos a la era digital, teniendo en cuenta que las disposiciones actuales se adoptaron en 1995, cuando menos del $1 \%$ de los europeos utilizaba Internet.

- Aumentar la confianza del consumidor en los servicios en línea facilitando una mejor información con respecto a los derechos y a la protección de datos mediante la introducción del derecho a la rectificación, al olvido y a la supresión, derecho a la portabilidad de datos y de oposición.

- Potenciar el mercado único digital reduciendo la fragmentación actual y las cargas administrativas, evitando las actuales divergencias en la aplicación de las normas de 1995 por parte de los Estados miembros y velar por que los derechos fundamentales a la protección de datos personales se apliquen de manera uniforme en todos los ámbitos de las actividades de la Unión Europea (UE).

\section{B) Sus ventajas (Parlamento Europeo 2015):}

1. Se dificultan las transferencias de datos a terceros países, sobre todo en casos de transferencias requeridas por tribunales o autoridades administrativas de países terceros. Si un país tercero solicita a una empresa (por ejemplo, una red social o un buscador) información personal procesada en la UE, dicha empresa deberá obtener el permiso de la autoridad nacional de protección de datos e informar a la persona en cuestión antes de enviar la información.

2. En su artículo 17, se sigue matizando el derecho al olvido, por el que cualquier persona podría solicitar que se borren sus datos si: 
- No se cumplen las normas de la UE.

- Los datos ya no son necesarios en relación con los fines para los que fueron recogidos o tratados.

- La persona retira o no da su consentimiento al almacenamiento de esa información.

La solicitud de este derecho quedará limitada cuando los datos hayan sido recopilados:

- Para ejercer la libertad de expresión.

- Para la investigación científica o histórica.

- Por motivos de salud pública.

- Con fines estadísticos.

3. Datos que revelen sanciones administrativas, fallos judiciales, presuntos delitos, creencias filosóficas y de orientación o identidad sexual no serán susceptibles de ser tratados.

4. Puesta en marcha del mecanismo 'One-stop-shop', por el cual cuando el proceso de datos se realice en más de un Estado miembro, las empresas solo tendrán que responder frente a una única autoridad de control, en lugar de 28, la cual monitorizará las actividades del responsable y el encargado del tratamiento, disminuyendo de esta manera la carga administrativa, aumentando la certeza legal y facilitando el desarrollo de negocios en la UE.

5. Consentimiento explícito y lenguaje claro. El consentimiento será entendido como una manifestación libre, concreta e informada de la voluntad del interesado, ya sea mediante una declaración o una clara acción afirmativa. Esto impediría, por ejemplo, prácticas como la de mantener marcada la casilla de 'Aceptar' en las políticas de privacidad.

\subsubsection{Carta de los Derechos Fundamentales de la Unión Europea}

En ella se reunieron por primera vez en un solo texto, todos los derechos que hasta ahora se repartían en distintos instrumentos legislativos como las legislaciones nacionales y los convenios internacionales del Consejo de Europa, de las Naciones Unidasy de la Organización Internacional del Trabajo.

Proclamada el 7 de diciembre de 2000 es un documento que "refuerza la protección de los derechos fundamentales, contribuyendo así a desarrollar el concepto de ciudadanía de la Unión y a crear un espacio de libertad, seguridad 
y justicia”(Unión Europea 2000).Se hizo jurídicamente vinculante con la entrada en vigor del Tratado de Lisboa el 1 de diciembre de 2009.

El derecho a la privacidad y a la protección de los datos de carácter personal, expuestos en los artículos 7 y 8 de esta Carta prevalecen, en principio, no sólo sobre el interés económico del gestor del motor de búsqueda, sino también sobre el interés público en disponer de la información de una persona, variando en función del papel que esa persona desempeñe en la vida pública, de la naturaleza de la información de que se trate y del carácter sensible para la vida privada de la persona afectada.

\subsubsection{Sentencia del Tribunal de Justicia de la Unión Europea, de 13 de mayo de 2014}

En el ámbito europeo sentó jurisprudencia la sentencia del Tribunal de Justicia de la Unión Europea (TJUE), de 13 de mayo de 2014, por la que "cualquier buscador en internet es responsable del tratamiento que aplique a los datos de carácter personalque aparecen en las páginas webs publicadas por terceros si son lesivas para un ciudadano y carecen de relevancia"(Tribunal de Justicia de la Unión Europea 2014).

Esta sentencia resolvió la demanda interpuesta por la Agencia Española de Protección de Datos y el ciudadano español Mario Costeja González contra Google Spain S.L. y Google Inc.

Pero vayamos a los antecedentes. El 19 de enero de 1998 en la edición en papel del periódico barcelonés La Vanguardia, apareció publicado, a instancia de la Dirección Provincial de la Tesoría General de la Seguridad Social de Barcelona, un anuncio sobre una subasta de bienes embargados, entre otros, a Mario Costeja González por una deuda contraída con la Seguridad Social (La Vanguardia 1998). Según el afectado, ese embargo se solucionó y la deuda la pagó, pero el problema volvió a ser actual cuando, una década después, La Vanguardia digitalizó su hemeroteca y la puso a texto completo en su web. Si se realizaba una búsqueda por el nombre y los apellidos de este ciudadano, Google ofrecía como resultado ese anuncio de embargo. Harto de que el buscador le presentara como un moroso, el Sr. Costeja inició en 2009 los trámites legales precisos para que su nombre desapareciera de Google.

La resolución (Tribunal de Justicia de la Unión Europea 2014)del litigio ordenó a Google adoptar las medidas necesarias para retirar de su índice los datos personales del Sr. Costeja González y a imposibilitar el acceso futuro a los mismos. El pronunciamiento del Tribunal también estableció límites, 
discriminando a quiénes se les puede solicitar la remoción de datos y a quiénes no. Los medios de comunicación, así como publicaciones literarias, periodísticas o artísticas, quedan excluidos. Las personas con vida pública, en tanto sea de interés general en 'recordar' lo que hicieron, también.

La sentencia es coherente y, en sí, no es mala para Google porque no le afecta a su negocio. En el contenido de la misma se realiza, además, un exhaustivo análisis de lo que hacen y dejan de hacer los motores de búsqueda y sobre si esta actividad puede considerarse o no tratamiento de datos. Se analiza el proceso que concluye en lo que se muestra en las páginas web como resultados de una búsqueda. En esos procesos ¿hay selección entre datos personales y el resto de información? Google dice que no y que, en todo caso, no es responsable de ese 'tratamiento de datos' ni ejerce control sobre los mismos. Pero a efectos prácticos de 'quien quiere saber algo' y de 'quien quiere que no se sepa algo' es lo mismo y es posible, entonces, reflexionar sobre lo que podemos y no podemos saber de los demás.

El diario barcelonés no se vio afectado por la sentencia en tanto sí es un medio de comunicación. Sin embargo, en este punto es preciso hacer una observación: La Vanguardia por un lado publica, pero por otro, en su edición digital también dispone de un buscador interno que... ¡hace lo mismo que Google!, es decir, recopila información de su hemeroteca -que pueden ser datos personales- y posteriormente, al buscar por el nombre de una persona presenta como resultado 'una visión estructurada de la información relativa a una persona'. Por tanto, no se ajusta a la todavía vigente Directiva europea 95/46/ $\mathrm{CE}$ de protección de datos. El buscador de un foro o de un blog personal probablemente también trabajen del mismo modo.

La persona interesada deberá presentar su solicitudvía 'online' al buscador (Yahoo, Bing, Google o cualquier otro), y este tiene que evaluar si es fundada. En caso de que el buscador no acceda a retirar la información, el afectado podrá dirigirse a la autoridad de control competente-en España, es la Agencia Española de Protección de Datos cuya web es http://www.agpd.es/-, o a los tribunales para que estos efectúen las pesquisas necesarias y, en su caso, ordenen al buscador la retirada de la información. Es decir, el Tribunal de Justicia de la Unión Europea, con dicha sentencia, abrió la puerta a un examen caso por caso de cada una de las reclamaciones presentadas a cualquier buscador.

Unos días después de la publicación de la sentencia, en concreto el 30 de mayo de 2014, Google crea un formulario(https://support.google.com/legal/ contact/lr_eudpa?product=websearch\&hl=es)para que, de acuerdo al fallo del Tribunal europeo, se pueda solicitar la retirada de los resultados de búsqueda 
que incluyan el nombre de una persona. Fue la aplicación práctica del 'derecho al olvido’ en Google.El primer día de su ejecución, Google recibe la estimable cifra de 12.000 solicitudes(Morel2014).Hasta el 9 de julio de 2015,el balance que el buscador ofrece en su informe de transparencia (http:// www.google.com/transparencyreport/removals/europeprivacy/) es el siguiente:

\begin{tabular}{|c|c|c|}
\cline { 2 - 3 } \multicolumn{1}{c|}{} & A NIVEL MUNDIAL & ESPAÑA \\
\hline Solicitudes que Google ha recibido & 281.638 & 26.425 \\
\hline $\begin{array}{l}\text { Total de URLs que Google ha evaluado para su } \\
\text { retirada }\end{array}$ & 1.024 .584 & 85.477 \\
\hline$\rightarrow$ URLs retiradas & $358.740\left(\mathrm{el} 41^{\prime} 3 \%\right)$ & $26.739\left(\mathrm{el} 366^{\prime} 4 \%\right)$ \\
\hline URLs NO retiradas & $510.065\left(\mathrm{el} 58^{\prime} 7 \%\right)$ & $46.791(\mathrm{el} 63,6 \%)$ \\
\hline
\end{tabular}

El 26 de junio de 2014, casi un mes después de la puesta en marcha del formulario de solicitud para bloquear resultados de búsqueda, Google inició la eliminación de resultados de búsqueda(Morel2014). Para que un ciudadanoconozca esta supresión de información en el resultado de una consulta,Google muestra este mensaje de advertencia en la parte inferior dela pantalla: 'Algunos resultados pueden haber sido suprimidos conforme a la ley europea sobre la protección de datos'. Lo que ha generadocuriosos servicios como 'Hidden from Google'-http://hiddenfromgoogle.com- o 'Forget.me'https://forget.me/-.

Otros buscadores como Bing o Yahoo (que funciona vía Bing) también se han visto obligados a aplicar la sentencia del TJUE y han tenido que efectuar una solución similara la de Google. En Bing/Yahoo el formulario de solicitud para bloquear resultados de búsqueda de acuerdo con la legislación europea está disponible en el siguiente enlace: https://www.bing.com/webmaster/ tools/eu-privacy-request? setlang=es.

\subsubsection{Grupo de Autoridades Europeas de Protección de Datos}

El Grupo de Autoridades Europeas de Protección de Datos, el llamado GT29, aprobó el 26 de noviembre de 2014 unas directrices sobre la aplicación de la sentencia del TJUE del 13 de mayo del mismo año relativa al 'derecho al olvido’ (Article 29 Data Protection Working Party2014). En ellas se analiza el pronunciamiento del Tribunal y desarrolla los criterios a tener en cuenta en la 
aplicación armonizada de la sentencia por parte de los Estados miembros de la UE, con independencia de que cada solicitud o petición de la ciudadanía debe ser analizada caso por caso. Algunos de los criterios son(Article 29 Data Protection Working Party 2014):

- Libertades de expresión e información. Los contenidos que sean de interés para el público por su naturaleza o por afectar a una figura pública no serán bloqueados.

- No se elimina información. El derecho de cancelación y oposición sólo afecta a los resultados obtenidos en las búsquedas hechas mediante el nombre de la persona y no implica que la página deba ser suprimida de los índices del buscador ni de la fuente original. En consecuencia, la información sigue intacta en la web original y accesible a través del buscador por cualquier otra palabra o término que no sea el nombre del afectado.

- Los motores de búsqueda están obligados a respetar los derechos de cancelación y de oposición reconocidos a toda la ciudadanía.

- Análisis individual. Teniendo en cuenta el impacto que la actividad de los buscadores tiene en los derechos a la privacidad y a la protección de los datos personales por cuanto permiten acceder desde cualquier lugar a múltiples informaciones personales, los derechos de los afectados prevalecen sobre el interés económico de los buscadores y sobre el interés de los internautas en acceder a información personal por ese cauce. Sin embargo, señala que es necesario realizar una evaluación caso por caso para alcanzar 'un justo equilibrio' entre los derechos e intereses. En cada caso, el resultado dependerá de la naturaleza y sensibilidad de los datos y del interés del público en acceder a una información, un interés en el que influye significativamente el papel que el afectado desempeñe en la vida pública.

- Ejercicio de derechos. Los ciudadanos se pueden dirigir directamente al motor de búsqueda sin necesidad de acudir previamente al sitio original. Los motores de búsqueda y los editores originales realizan dos procesamientos de datos diferenciados, con legitimaciones diferentes y también con un impacto diferente sobre la privacidad de las personas. Por eso puede suceder, y de hecho sucede con frecuencia, que el contenido que publica el editor siga siendo legal con el paso del tiempo mientras que la difusión 
universal que realiza el buscador, sumado a la información adicional que facilita sobre el mismo individuo cuando se busca por su nombre, tiene un impacto desproporcionado sobre su privacidad.

- Buscadores internos. Los buscadores propios incluidos en las webs de diferentes páginas o medios de comunicación no están afectados por la sentencia del Tribunal Europeo de Justicia. Estos buscadores internos sólo recuperan la información contenida en páginas web específicas y, además, no permiten establecer un perfil completo de la persona afectada, algo que sí permiten los motores de búsqueda.

- Ámbito de aplicación. La exclusión debe también ser eficaz en todos los dominios relevantes europeos y de otros países, incluidos los '.com', que se puedan consultar desde el territorio de la Unión Europea.

- Política de avisos. La práctica de algunos buscadores de informar a los usuarios de que la lista de resultados puede no estar completa como consecuencia de la aplicación del derecho europeo no encuentra fundamento en ninguna exigencia normativa. Esta práctica sólo puede ser aceptable si la información se ofrece de tal manera que los usuarios no puedan deducir, en ningún caso, que una persona concreta ha solicitado la retirada de ciertos resultados asociados a su nombre.

- Transparencia. El GT29 insta a los buscadores a publicar los criterios de exclusión que aplican y a facilitar estadísticas sobre los tipos de casos en los que han aceptado o rechazado las correspondientes solicitudes.

La Unión Europea no quiere que el 'derecho al olvido' se limite al marco fronterizo de sus países miembros y por ello el GT29 ha instado a Google a que también lo aplique en google.com,el sitio web de Google en EE.UU. y no sujeto, por tanto, al control que impone al buscador la sentencia del Tribunal de Justicia de la Unión Europea( Gibbs 2014). Sin embargo, la realidad es que hasta el momento se limita a las webs europeas. De hecho, los formularios tanto de Google como de Bing ya lo exponen claramente: "Solicitud de retirada de resultados de búsqueda en virtud de la normativa de protección de datos europea", se titula el formulario de Google-disponible en: https:// support.google.com/legal/contact/lr_eudpa?product=websearch\&hl=es-; y "Formulario de solicitud para bloquear resultados de búsqueda de acuerdo con la legislación europea”, el de Bing- disponible en: https://www.bing.com/ 
webmaster/tools/eu-privacy-request? setlang=es-.

Aunque mucho menos famoso que Google o Bing y sin fines de lucro, otro gigante de los laberintos de la red es Internet Archive-https://archive.org/ web/-. Su infraestructura queda fuera de la jurisdicción europea y tiene como misión recabar datos de la red, lo cual incluirá inevitablemente datos personales que estén publicados justamente para 'evitar' el olvido.

\subsection{El Consejo Asesor de Google sobre Derecho al olvido}

Con el fin de cumplir el mandato de la sentencia del Tribunal Europeo de Justicia, Google creó este Consejo Asesor, del que aporta información, quizá en un afán de completa transparencia en https://www.google.com/ advisorycouncil/.

Formado por 10 especialistas ajenos a la compañía estadounidense, pero costeados por ella misma,su misión es el asesoramiento en las complejas cuestiones que entran en juego cuando convergen el derecho a la información y el derecho a la privacidad. Para ello, el Consejo Asesor ha estudiado las guías aportadas por las Agencias de Protección de Datos Europeas, las opiniones vertidas por un gran número de expertos que participaron en varias reuniones celebradas en diversas ciudades europeas entre septiembre y noviembre de 2014, así como las procedentes de miles de comentarios recibidos a través de la web.

Y en febrero de este año publicó un informe.Algunas de las conclusiones que en él se exponen son las siguientes (Consejo Asesor de Google sobre Derecho al olvido 2015):

- El derecho a salir de Google no debe ser universal.

- Se divide el tipo de información para sugerir al buscador que no toda la información 'pesa' lo mismo a la hora de eliminarla.

- Es recomendable conservar:

- Información sobre salud, consumo, arte o ciencia.

- Información general sobre la situación financiera de un ciudadano.

- Información sensible —origen racial, opiniones políticas, credo - de personajes públicos cuando tenga relación con el rol que juega en la sociedad.

- Enlaces con declaraciones públicas de políticos.

- Contenidos relacionados con un debate de interés público. 
- Es recomendable eliminar:

- Datos falsos sobre una persona.

- Enlaces sobre la vida íntima o sexual de un ciudadano.

- Información como números de teléfonos, direcciones postales y documentación administrativa del ciudadano (número de pasaporte, permiso de conducir, tarjeta sanitaria, etc.).

\subsection{El 'derecho al olvido’ en España}

Considerando que todo el acervo legistativo que se desarrolle en la Unión Europea -y que hemos tratado anteriormente- tiene aplicación directa en cualquiera de sus países miembros, sólo vamos a citar algunas peculiaridades propiamente españolas.

La Constitución Española de 1978 en sus artículos 18.1 y 20.4 garantiza el derecho al honor, a la intimidad personal y familiar y a la propia imagen, que constituyen 'límites' a la libertad de expresión e información. Y el artículo 18.4 establece que "la ley limitará el uso de la informática para garantizar estos derechos"(España1978)que, aunque están vinculados con el derecho al olvido en internet, sería muy aventurero afirmar que nuestro texto constitucional sí que lo expone explícita e implícitamente. Por otro lado y como es evidente, en el momento en que se redactó nuestra Constitución, Internet a nivel de usuario todavía no existía.

La Ley orgánica 15/1999, de 13 de diciembre, de protección de datos de carácter personal (España 1999)y su reglamento de desarrollo (España 2008) establecieron las bases para comenzar a regular los vacíos legales con los que se encontraba el ciudadano español en sus demandas internautas por proteger su privacidad e intimidad. Aunque en ninguno todavía no se cita expresamente el derecho al olvido, su Título III se ocupa de los derechos de los ciudadanos en el tratamiento de sus datos personales, incluyendo el derecho a tener conocimiento de todos los datos sobre uno mismo que se encuentran recogidos en Internet y su tratamiento, mediante los derechos de acceso, rectificación, cancelación y oposición.

El ciudadano español para ejercer su 'derecho al olvido' en Internet tiene que dirigirse al buscador. ¿Y qué pasa si el buscador decide no eliminar el enlace? Si la petición no es favorable, el ciudadano tiene el derecho deinvocar la tutela de la Agencia Española de Protección de Datos-https://www.agpd.es/.Creada en 1993(España1993), es el principal organismo donde la ciudadanía 
debe dirigirse para:

- Solicitar la cancelación de datos de páginas webs.

- Impedir que sean difundidos por buscadores, redes sociales, blogs, etc., si se estima que vulnera el respeto de la dignidad personal.

Las reclamaciones que principalmente gestionan son de dos tipos:

- Investigaciones. La mayoría tenía que ver con la difusión no autorizada de datos personales.

- Peticiones. Afectan a la recuperación por buscadores de datos en boletines oficiales o medios de comunicación digitales sobre sanciones administrativas ya cumplidas, edictos de deudas vencidas o datos de víctimas de violencia doméstica.

También han surgido iniciativas privadas que ofrecen asesoramiento en la privacidad y protección de datos personales, como la Asociación Profesional Española de Privacidad-http://www.apep.es/-; además de propuestas empresariales cuyos servicios ofertados son la eliminación de datos del ciberespacio.Entre otras:'Eprivacidad'-http://www.eprivacidad.es/-y 'Salir de Internet'-http://www.salirdeinternet.com/-.

Desde la sentencia de 13 de mayo de 2014 del Tribunal de Justicia de la Unión Europea y hasta el 12 de julio de 2015, la Audiencia Nacional ha resuelto sesenta y nueve recursos relativos al derecho al olvido en Internet, los primeros en los que aplica el criterio establecido por la sentencia europea.En cincuenta y dos de los recursos, la Audiencia da la razón a los particulares, mientras que en los otros diecisiete estima los argumentos del buscador (Audiencia Nacional 2015).

\section{EL OLVIDO COMO AMENAZA}

La pérdida de memoria como enfermedad mental pasa por varias definiciones médicas y psicológicas extensas de explicar. Nos centraremos en la Enfermedad de Alzheimer (también denominada demencia senil, enfermedad neurodegenerativa que se manifiesta como deterioro cognitivo y se caracteriza por una pérdida de la memoria inmediata) y la amnesia (afección de diferentes causas orgánicas y funcionales en la cual la memoria se ve alterada, con la imposibilidad de recuperar información almacenada anteriormente). 
Según Javier Olazaránet al., (2010)existen dos tipos de tratamientos para las enfermedades donde habita el olvido: el farmacológico y las terapias sin fármacos. Para este segundo caso, pueden ser varios grupos de profesionales (terapeutas ocupacionales, psicólogos, fisioterapeutas...), incluso familiares de los pacientes, quienes pueden llevarlas a cabo, en un intento de paliar los efectos de estos procesos, a falta de tratamientos curativos y ya aplicadas desde 1960 .

Dentro de estas terapias no farmacológicas - la lista es ampliasegún la Clasificación del Grupo Internacional de Trabajo de Terapias No Farmacológicas (INPTP), liderado desde la Fundación Maria Wolff(Fundación Centro de Investigación Enfermedades Neurológicas 2013, 2014)-ynos centraremos principalmente en dos: laestimulación cognitiva (facilitación general y temática de operaciones cognitivas basada en la evocación, la relación y el procesamiento) y la reminiscencia (elaboración cognitiva y afectiva a partir de acontecimientos o experiencias del pasado remoto (acontecimientos vividos, hechos del pasado, canciones antiguas...), porque ambas llevan al encuentro con la memoria y los recuerdos anteriores.

La puesta en práctica de tales actividades es mediante talleres de memoria donde se trabaja la evocación de los nombres de familiares, lugares, alimentos, etc. También con fotografías personales, a modo de juego, que una vez mostradas y visualizadas se solicita al paciente el nombre de las personas que aparecen, nombre de las prendas de ropa que llevaba, nombre del lugar donde se tomó la foto, descripción de ese lugar, reconocer otros elementos que aparecen y después se retiran las fotografías o imágenes de la vista. También se le pide al paciente que recuerde datos que aparecían en la fotografía: personas, ropa que llevaban, lugar y otros datos (si era por la mañana, por la noche, etc.).

Además, como ya se ha visto, las estrategias para almacenar el pasado son múltiples, y todas llevan el mismo camino y van dirigidas al mismo destino: hacer frente al olvido.

Si tan importante es mantener los recuerdos del pasado, centrándonos en la tecnología actual, ésta puede llegar a ser cómplice de que el presente transcurra tan rápido que no dé tiempo a que se convierta en pasado. A nivel de gran consumo de la tecnología en las empresas, las medidas de preservación están más controladas y reguladas por la existencia de intereses generados de la propia actividad empresarial; pero en el nivel más personal, el uso de la tecnología para correos, mensajes e imágenes es todo demasiado veloz y la no conservación depende de la capacidad de memoria de los dispositivos con los que nos comunicamos y de los que ya dependemos. 
¿Qué será de todos nuestros recuerdos visuales? ¿Cómo serán los talleres de memoria si no tenemos la precaución de revelar fotografías, todas alojadas en dispositivos electrónicos que queden obsoletos pasados unos años, además de estar expuestos a virus que exterminen nuestro pasado?

Nuestros recuerdos visuales quedan, por tanto, a la deriva de la tecnología y la buena disposición de cada uno de nosotros para volver al revelado de fotografías y su custodia en álbumes ordenados, o en cajas de cartón con una pegatina indicando: fotos, porque éstas son fieles reflejos del pasado, una forma de revivir acontecimientos en cualquier presente y un refugio de recuerdos para el futuro.

Actualmente, el espacio digital, "viene a cumplir las mismas funciones que sus predecesores pero, además, nos revela mayores prestaciones para la imagen, y al tiempo integra una serie de propiedades que recogen y amplían a la vez las aportaciones realizadas por los soportes precedentes"(Pantoja Chaves 2007).

En definitiva, tal y como señala Martín Nieto (2005) se trata de tener muy presente el uso de la fotografía como fuente para la memoria, como recurso para el no olvido, con el cuidado y la precaución de salvar -si no todas, algunas - de las miles de fotografías que se conservan en móviles, tabletas y cámaras de fotos digitales, que a lo que más llegan es a pasar a un ordenador o al almacenaje virtual. Una fotografía para el recuerdo hay que tocarla, pues "el tacto es otro de los estímulos de la terapia no farmacológica para combatir el olvido"(Fundación Centro de Investigación Enfermedades Neurológicas 2014) $\mathrm{y}$ aunque las fotos puedan imprimirse en casa, dos inconvenientes frenan la impresión:

- La impresora láser, el papel y tinta especiales suponen un coste muy elevado.

- Las copias en papel fotográfico para impresoras de chorro de tinta tienen una vida útil de unos 5 años, a menos que se adquiera tinta y papel especial.

Ambos motivos retrasan o evitan el revelado de todas las fotos de nuestra vida, no como antaño, que un carrete de 36 exposiciones a color era un lujo porque, además, cada fotografía añadía la incertidumbre del resultado final, algo que podía llevar meses de espera, lo que sobrevaloraba el recuerdo. Sin embargo, hoy día, la inmediatez, el exceso de fotos personales, la circulación indiscriminada de ellas y la custodia no adecuada, han variado ese valor del recuerdo, y todos, alguna vez, hemos lamentado haber perdido fotos, momentos que no se repetirán, por... ¿confiados o descuidados? 


\section{CONCLUSIONES}

\subsection{El olvido como necesidad}

- En la Sociedad del Conocimiento en la que estamos se producen conflictos en los que late la tensión entre lo que se puede o no se puede saber de los demás: privacidad e intimidad versus derecho a saber. $\mathrm{Y}$ en esta dicotomía, el ciudadano está planteando el derecho a que se eliminen datos que los buscadores encuentran y que puede considerar lesivos de su intimidad o que afecten a su imagen cuando los mismos no sean de interés público.

- En este conflicto, los buscadores en general y Google en particular han defendido su posición considerándose, de alguna manera, ajenos a los contenidos existentes en las fuentes de la información, mientras que el Tribunal de Justicia de la Unión Europea ha considerado que no son tan ajenos y les ha impuesto una serie de obligaciones.

- Esto nos conduce a pensar si de lo que se trata es de que la información desaparezca del lugar en la que está, o, en cambio, si es necesario generar un mecanismo para que resulte inencontrable. Estamos, más bien, en el segundo supuesto. Es como si el buscador de Internet fuera un gran telescopio que muestra lo que hay ahora, y por imperativo de una restricción vinculada con la privacidad, se le obligara a ocultar la información en cuestión.

- Pero, ¿quién gana con el derecho al olvido? Cuesta imaginar una ingente masa de ciudadanos anónimos, antes desprotegidos por el acecho del recuerdo, que ahora recurrirán masivamente a los tribunales para hacer valer sus olvidos. Es probable que el grupo de urgidos demandantes del olvido digital no difiera demasiado de aquel que por su voluntariamente elegido alto nivel de exposición -y elevado nivel de recursos- suele mantener duras controversias judiciales con los medios de comunicación en pugna por el control del discurso sobre sí mismos: políticos, famosos de la prensa rosa, modelos, deportistas de alta competición, banqueros y ladrones de guante blanco, entre otros.

-El derecho al olvido no es un derecho absoluto. Cuando entra en 'conflicto' con otros derechos, la autoridad reguladora (en el caso de España, la Agencia Española de Protección de Datos) pondera cada caso según los criterios de exactitud, relevancia pública y obsolescencia. 
- Es plenamente compatible con la libertad de expresión y con el derecho a recibir información.

-El derecho a la protección de datos de la ciudadanía prevalece, con carácter general, sobre el mero interés económico de los buscadores.

El derecho al olvido no es un intento de reescribir la historia ni de alterar la hemeroteca. Cuando la publicación original es legítima (un periódico, por ejemplo), no comporta el derecho a borrar esa información del soporte original. Sólo se elimina de los resultados de los buscadores para que los datos lesivos no sean perpetuos en la memoria digital.

\subsection{El olvido como amenaza}

La memoria en las personas es fundamental para poder seguir formando parte de la sociedad; por el contrario, su pérdida deja a los individuos aislados.

Aunque la recuperación de algunas enfermedades donde la memoria queda dañada es casi imposible, sí hay métodos para retrasar el deterioro cognitivo: aferrarse al recuerdo con elementos del pasado individual. Las fotografías juegan un papel importante.

Si bien la tecnología ayuda a almacenar miles de imágenes, también la pérdida o cambio de la tecnología puede afectar a la pérdida de recuerdos personales irrecuperables.

En prevención al deterioro memorístico que cualquiera puede sufrir, la correcta conservación de los recuerdos personales son una garantía para hacer frente a la situación.

\section{A modo de Epílogo:}

Si Borges hubiera conocido Internet y su capacidad de abarcar todo el conocimiento en cualquier momento, habría sentido que se hacía realidad el personaje de su cuento Funes el memorioso, aquel joven de 19 años capaz de no olvidar todo aquello que vivía, leía y soñaba (Borges 1993). 


\section{4.-BIBLIOGRAFÍA}

ARTICLE 29 DATA PROTECTION WORKING PARTY, 2014. Guidelines on the implementation of the Court of Justice of the European Union Judgment on "Google Spain and Inc v. Agencia Española de Protección de Datos (AEPD) and Mario Costeja González" :C-131/121, WP 225, adopted on 26 November 2014[en línea].Brussels: European Commission, Directorate General Justice[Consulta: 10 julio 2015]. Disponible en: http://www.cr-international.com/38571.html.

AUDIENCIA NACIONAL, 2015. Jurisprudencia[en línea]. Madrid: Consejo General del Poder Judicial. [Consulta: 12 julio 2015]. Disponible en:

http://www.poderjudicial.es/cgpj/es/Poder-Judicial/Audiencia-Nacional/ Jurisprudencia/Jurisprudencia-de-la-AN.

BORGES, J. L., 1993. Funes el memorioso. En: Artificios. Madrid: Alianza, pp. 7-18. ISBN 8420646067.

CERNUDA, L., 1982. La realidad y el deseo. Madrid: Alianza. ISBN 8470394029.

CESDEA SOLUCIONES JURIDICAS, 2015.Diccionario jurídico [en línea]. Córdoba: CESDEA. [Consulta: 28 mayo 2015]. Disponible en: http://diccionariojuridico.net/.

CONSEJO ASESOR DE GOOGLE SOBRE DERECHO AL OLVIDO, 2015. Informe [en línea]. Mountain View(California): Google. [Consulta: 14 mayo 2015]. Disponible en: https://drive.google.com/a/google.com/file/ d/oB1UgZshetMd4cEI3SjlvVohNbDA/view?pli=1.

CORTES GABAUDÁN, Francisco (coord.), 2011. Dicciomed.eusal.es.: diccionario médico-biológico, histórico y etimológico [en línea]. Salamanca: Ediciones Universidad. [Consulta: 3 mayo 2015]. Disponible en:http://dicciomed.eusal.es/palabra/amnesia.

DUVA, J., 1989. El golpe soñado. En: EL PAÍ́s [en línea]. Madrid: Grupo Prisa. [Consulta: 9 mayo 2015]. Disponible en: http://elpais.com/diario/1989/08/04/ madrid/618233056_850215.html.

'El Dioni' roba un furgón lleno de dinero, 1989.En: Antena3.com[en línea]. Madrid: Atresmedia. [Consulta: 11 mayo 2015]. Disponible en: http://www.antena3.com/ especiales/noticias/en-un-mes-de/julio/dioni-roba-furgon-llenodinero_2011062700092.html.

Enciclopedia jurídica [en línea] [Consulta: 10 julio 2015]. Disponible en: http:// www.enciclopedia-juridica.biz 14.com/inicio-enciclopedia-diccionario-juridico.html.

Enciclopedia jurídica online[en línea]. Dover, México, Bogotá: International Lawyers Network. [Consulta: 27 mayo 2015]. Disponible en: http://leyderecho.org/derecho-alolvido/.

ESPAÑA, 1978. Constitución Española. Boletín Oficial del Estado, 311, 29 de diciembre de 1978, 29315-29339. ISSN 0212-033X.

ESPAÑA, 1985. Convenio 108 del Consejo de Europa para la protección de las personas respecto al tratamiento automatizado de datos de carácter personal (versión en castellano). Boletín Oficial del Estado, 274, 15 de noviembre, 3600036004. ISSN 0212-033X. 
ESPAÑA, 1993. Real Decreto 428/1993, de 26 de marzo, por el que se aprueba el Estatuto de la Agencia Española de Protección de Datos.Boletín Oficial del Estado, 106, 4 de mayo, 13244-13250. ISSN 0212-033X.

ESPAÑA, 1999. Ley orgánica 15/1999, de 13 de diciembre, de protección de datos de carácter personal.Boletín Oficial del Estado, 298, 14 diciembre 1999, 43088-43099. ISSN 0212-033X.

ESPAÑA, 2008. Real Decreto 1720/2007, de 21 de diciembre, por el que se aprueba el Reglamento de desarrollo de la Ley Orgánica 15/1999, de 13 de diciembre, de protección de datos de carácter personal.Boletín Oficial del Estado, 17, 19 de enero, 4103-4136. ISSN 0212-033X.

FERNÁNDEZ MARTÍNEZ, J. M. coord., 2012. Diccionario jurídico. Cizur Menor: Aranzadi. ISBN 9788490141885.

FUNDACIÓN CENTRO DE INVESTIGACIÓN ENFERMEDADES NEUROLÓGICAS, 2013.Terapias no farmacológicas para la enfermedad de Alzhéimer.En: Blog de la Fundación CIEN [en línea].[Consulta: 30 mayo 2015]. Disponible en: http://fundacioncien.es/blog/index.php/2013/05/terapias-nofarmacologicas-para-la-enfermedad-de-alzheimer/.

FUNDACIÓN CENTRO DE INVESTIGACIÓN ENFERMEDADES NEUROLÓGICAS, 2014.Terapias no farmacológicas en demencias. En: Blog de la Fundación CIEN [en línea].[Consulta: 31 mayo 2015]. Disponible en: http:// fundacioncien.es/blog/index.php/2014/05/terapias-no-farmacologicas-en-demencias/.

FUNDACIÓN TOMÁS MORO, 1997. Diccionario jurídico Espasa. Madrid: Espasa Calpe. ISBN 8423959880.

---GIBBS, S., 2014. EU to Google: expand 'right to be forgotten' to Google.com. En:The Guardian [en línea]. Londres: Guardian Media Group[Consulta: 11 julio 2015]. Disponible en:

http://www.theguardian.com/technology/2014/nov/27/eu-to-google-expand-right-tobe-forgotten-to-googlecom.

LA VANGUARDIA, 1998. Hemeroteca[en línea], 19 de enero. Barcelona: La Vanguardia Ediciones. [Consulta:16 mayo 2015]. Disponible en: http:// hemeroteca.lavanguardia.com/preview/1998/01/19/pagina-23/33842001/pdf.html.

MARTÍN NIETO, E., 2005. El valor de la fotografía. Antropología e imagen [en línea]. Gazeta de Antropología, 21. Disponible en: http://www.ugr.es/ p pwlac/ G21_04Eva_Martin_Nieto.html.

MONTOLIU, P.,1989. Desaparece el conductor de un furgón blindado con los 320 millones de pesetas que transportaba. En: EL PAÍS [en línea]. Madrid: Grupo Prisa. [Consulta: 8 mayo 2015]. Disponible en:http://elpais.com/diario/1989/07/30/ madrid/617801056_850215.html.

MOREL, J., 2014. Google y el olvido: crónica de un trimestre agitado. En:El Diario.es [en línea]. Madrid: Diario de Prensa Digital [Consulta: 22 mayo 2015].Disponible en: http://www.eldiario.es/hojaderouter/ilegales/googlederecho_al_olvido-cronologia_6_299630061.html.

OLAZARÁN, J. et al., 2010.Eficaciade las terapias no farmacológicas en la enfermedad 
de Alzheimer: una revisión sistemática.Dementia and geriatric cognitive disorders, 30, 161-178. ISSN 1420-8008.

PANTOJA CHAVES, A., 2007. La sociedad de la imagen. La fotografía como recurso para la memoria histórica [en línea]. En: I Encuentro de Jóvenes Investigadores en Historia Contemporánea de la Asociación de Historia Contemporánea. 26-28 septiembre. Zaragoza: Asociación de Historia Contemporánea [Consulta: 18 junio 2015]. Disponible en:http://ifc.dpz.es/recursos/publicaciones/27/15/55.antoniopantoja.pdf.

PARLAMENTO EUROPEO, 2014. Resolución legislativa del Parlamento Europeo, de 12 de marzo de 2014, sobre la propuesta de Reglamento del Parlamento Europeo y del Consejo relativo a la protección de las personas físicas en lo que respecta al tratamiento de datos personales y a la libre circulación de estos datos (Reglamento general de protección de datos $)(\operatorname{COM}(2012) 0011-\mathrm{C} 7-0025 / 2012-$ 2012/0011 (COD)).En:Parlamento Europeo. Documentos[en línea]. Estrasburgo: Parlamento Europeo [Consulta: 22 junio 2015]. Disponible en: http://www.europarl.europa.eu/ sides / get Doc.do? pubRef=-// E P / / T EX T + T A + P 7 - T A - 2014 $\mathrm{O} 212+\mathrm{O}+\mathrm{DOC}+\mathrm{XML}+\mathrm{Vo} / /$ ES\&language $=\mathrm{ES}$.

PARLAMENTO EUROPEO, 2015.Data protection: Parliament's negotiators welcome Council negotiating brief.En:Parlamento Europeo en portada. Noticias [en línea]. Estrasburgo: Parlamento Europeo [Consulta: 15 junio 2015]. Disponible en:http:// www.europarl.europa.eu/news/es/news-room/content/20150615IPR66464/html/Data -protection-Parliament\%E2\%80\%99s-negotiators-welcome-Council-negotiating-brief.

REAL ACADEMIA ESPAÑOLA (RAE). Diccionario de la lengua española[en línea]. Madrid: RAE. [Consulta: 1 mayo 2015]. Disponible en: http://lema.rae.es/drae/? val=OLVIDO.

TRIBUNAL DE JUSTICIA DE LA UNIÓN EUROPEA, 2014. Sentencia del Tribunal de Justicia de la Unión Europea, de 13 de mayo de 2014, Google Spain SL y Google Inc. contra Agencia Española de Protección de Datos (AEPD) y Mario Costeja González. En: Eur-Lex [en línea]. Luxemburgo: Oficina de Publicaciones de la Unión Europea. [Consulta: 7 mayo 2015]. Disponible en:http://eur-lex.europa.eu/legal-content/ES/ TXT/?qid=1430995149864\&uri=CELEX:62012CJ013 1

UNIÓN EUROPEA, 1995. Directiva 95/46/CE del Parlamento Europeo y del Consejo, de 24 de octubre de 1995, relativa a la protección de las personas físicas en lo que respecta al tratamiento de datos personales y a la libre circulación de estos datos. Diario Oficial de las Comunidades Europeas, Serie L, 281, 23 de noviembre, 31-50. ISSN 1012-9200.

UNIÓN EUROPEA, 2000. Carta de los Derechos Fundamentales de la Unión Europea. Diario Oficial de las Comunidades Europeas, Serie C, 364, 18 de diciembre, 1-22. ISSN 1012-9200.

UNIVERSIDAD NACIONAL DE EDUCACIÓN A DISTANCIA (UNED), 2014. Diccionario jurídico de derecho [en línea]. Madrid: UNED. [Consulta: 28 mayo 2015]. Disponible en: http://www.uned-derecho.com/diccionario/.

VILLA-REAL MOLINA, R. y ARCO TORRES, M. A., 1999. Diccionario de términos jurídicos. Granada: Comares. ISBN 848151828X.

ZAMBRANO, M., 2005. Séneca. $3^{\text {a }}$ ed. Madrid: Siruela. ISBN 8478442243. 
MEI, II, Vol. 6, no 10, pág. 108

\section{5.- NOTAS}

1- Título tomado del poema de Luis Cernuda, poeta de la Generación del 27, que publicó en su libro La realidad y el deseo.

2-campopuerta@gmail.com

3-rafa_jaros@yahoo.es 